\title{
Surveillance of Extrapulmonary Nontuberculous Mycobacteria Infections, Oregon, USA, 2007-2012
}

\author{
Emily Henkle, Katrina Hedberg, Sean D. Schafer, Kevin L. Winthrop
}

Limited data are available describing extrapulmonary nontuberculous mycobacteria (NTM) infections in the general population. We describe results from statewide population-based laboratory surveillance in Oregon, USA, during 2007-2012. We defined a case of extrapulmonary NTM infection as $\geq 1$ isolate from skin/soft tissue, disseminated sites, lymph node, joint, or other sites. The annual incidence of extrapulmonary NTM infection (other than Mycobacterium gordonae) was stable, averaging 1.5 cases/100,000 population. Median age of the 334 patients was 51 years, and $53 \%$ of patients were female. Half of cases were caused by $M$. avium complex, but rapid-growing NTM species accounted for one third of cases. Most extrapulmonary NTM infections are skin/soft tissue. Compared with pulmonary NTM infection, more extrapulmonary infections are caused by rapid-growing NTM species. The designation of NTM as a reportable disease in Oregon in 2014 will result in better detection of changes in the incidence and patterns of disease in the future.

Tontuberculous mycobacteria (NTM) are ubiquitous 1 in water and soil and are a cause of opportunistic pulmonary and extrapulmonary infections. Extrapulmonary manifestations include disseminated, skin, joint, and lymph node infections. Extrapulmonary NTM infections are typically sporadic but may be associated with nosocomial outbreaks (1), clinical procedures (2), or nail salon pedicures (3). Treatment is species dependent, typically consisting of 3-6 months of multidrug antimicrobial therapy (4). Although numerous case series are found in the literature, few data exist to describe the populationbased epidemiology of extrapulmonary NTM infections. Recent studies have shown an increase in the prevalence and incidence of pulmonary infections $(5,6)$. We describe the results of a statewide laboratory surveillance study in Oregon, USA, that identified all patients from whom NTM was isolated from extrapulmonary sites during 2007-2012. We report characteristics of patients with

Author affiliations: Oregon Public Health Division, Portland, Oregon, USA (E. Henkle, K. Hedberg, S.D. Schafer); Oregon Health and Science University, Portland (E. Henkle, K.L. Winthrop)

DOI: https://doi.org/10.3201/eid2310.170845 extrapulmonary NTM infection by species and site and calculate the annual incidence over the study period.

\section{Methods}

Methods have been described elsewhere (5). In brief, we requested positive NTM culture results from January 1, 2007, through December 31, 2012, from all 17 laboratories that perform acid-fast bacillus culture in Oregon or are used as reference laboratories. We excluded $M$. tuberculosis and $M$. bovis. Additional data for each positive culture included patient name, age at collection or date of birth, address or county and ZIP code of residence, species isolated, date of collection, and body source of isolate. One laboratory in central Oregon was unable to provide enough information to assign a state of residence. Given a low likelihood of these patients coming from out of state (this lab is $>120$ miles from Washington, Idaho, or California), however, we considered all these patients to be Oregon residents. After we identified the patients, we linked patients with extrapulmonary isolates to the state HIV database to identify any who were infected with HIV.

\section{Case Definition}

We defined an extrapulmonary NTM infection case as having $\geq 1$ isolates from skin/soft tissue (wound, abscess, tissue, or exit catheter); disseminated sites (blood, bone marrow, cerebrospinal fluid, pericardial fluid, or peritoneal fluid); lymph node (lymph node or neck abscess); joint (synovial or joint fluid); or other (urine, eye, sinus, or nasopharyngeal). We excluded isolates from an unknown source or from feces, saliva, or gastric sites. M. gordonae was reported but is considered nonpathogenic, so we excluded it from estimates of disease incidence. Rapid-growing mycobacteria (RGM) species include $M$. chelonaelabscessus complex, M. fortuitum, and M. chelonae (4); we included these in the analysis.

\section{Statistical Analysis}

We described patients by age, sex, and species isolated overall and by source of specimen. We considered extrapulmonary cases to be incident at the time of isolation, given that treatment for extrapulmonary infections is typically in the range of weeks to several months and the cure rate is high (4). We calculated the annual incidence as the number 
of new cases in a given year divided by the midyear population using population data from the Portland State University Population Research Center (7) and report the average annual incidence and standard 95\% CIs for 2007-2012. We used Poisson models using a log link to estimate the overall incidence rate trend over the study period. We imported all data into SAS version 9.3 (SAS Institute Inc., Cary, NC) for analysis. The study was considered to be public health practice (nonresearch) by the Oregon Health Authority and was conducted under Oregon Administrative Rule 333019-0005 (Conduct of Special Studies).

\section{Results}

\section{Patient Characteristics and Species Isolated}

We identified 334 patients with extrapulmonary isolates, including 1 patient with 2 distinct infections. Overall, 176 $(53 \%)$ patients were female, and the median age was 50 years (range $0.8-92$ years) (Table). Half $(\mathrm{n}=167,50 \%)$ of patients had M. avium/intracellulare complex (MAC) infection, 129 (38.6\%) RGM infection, and $21(6.3 \%) M$. marinum infection.

\section{Results by Site of Infection}

Among the 334 extrapulmonary NTM infections, 197 $(59.0 \%)$ were skin/soft tissue, $57(17.1 \%)$ were disseminated, $28(8.4 \%)$ were lymph node, 14 (4.2\%) were joint, and $38(11.4 \%)$ were other (Table). The overall species distribution was 50\% MAC, $22.8 \%$ M. chelonae/abscessus complex or M. chelonae, $9.6 \%$ M. fortuitum, and $6.3 \%$ M. marinum. We identified an additional 13 patients with M. gordonae isolates. Patients with skin/soft tissue infections were more commonly female (58\%), whereas disseminated infections occurred predominantly in male patients $(67 \%)$; of these, $79 \%$ of patients had MAC infection. The median age of patients with disseminated infections was 41 years, and $60 \%$ of these infections occurred in HIV-positive patients. Lymph node infections were $82.1 \%$ MAC; $18 \%$ of these patients were HIV positive. Among $23 \mathrm{HIV}$-negative patients with lymph node infections, 10 (44\%) were $<5$ years of age. Only 14 joint infections were reported; these patients had a median age of 70 years, and $64.3 \%$ of infections were caused by MAC. Of other infections, $52.6 \%$ were caused by MAC and $31.6 \%$ by $M$. chelonaelabscessus complex or M. chelonae.

\section{Estimates of Annual Incidence}

The average annual incidence of extrapulmonary NTM infection during 2007-2012 was 1.5 (95\% CI 1.1-1.8) cases $/ 100,000$ population. The incidence was 1.4 (CI 1.0 1.8) cases $/ 100,000$ population in 2007 , peaked at 1.7 (CI 1.3-2.1)/100,000 in 2009, and decreased to 1.3 (CI 1.0 1.7)/100,000 in 2012 (Figure). The Poisson estimate of change in annual incidence was not significant, at $-1.8 \%$ (95\% CI -0.08 to $0.04 ; p=0.6$ ). The incidence by site of isolation was 0.9 cases $/ 100,000$ population for skin/soft tissue, $0.2 / 100,000$ for disseminated, $0.2 / 100,000$ for lymph, $0.1 / 100,000$ for joint, and $0.2 / 100,000$ other. The average annual incidence was 0.7 cases/100,000 population for MAC, $0.2 / 100,000$ for

\begin{tabular}{|c|c|c|c|c|c|c|}
\hline \multirow[b]{2}{*}{ Category } & \multicolumn{5}{|c|}{ Infection site $\dagger$} & \multirow[b]{2}{*}{ Total, $\mathrm{N}=334$} \\
\hline & $\begin{array}{c}\text { Skin/soft tissue, } \\
n=197\end{array}$ & $\begin{array}{l}\text { Disseminated, } \\
\mathrm{n}=57\end{array}$ & $\begin{array}{l}\text { Lymph node, } \\
n=28\end{array}$ & $\begin{array}{l}\text { Joint, } \\
\mathrm{n}=14\end{array}$ & Other, $n=38$ & \\
\hline Annual incidence $/ 100,000$ population & 0.9 & 0.2 & 0.1 & 0.1 & 0.2 & 1.5 \\
\hline \multicolumn{7}{|l|}{$\begin{array}{l}\text { Patient demographics } \\
\text { Sex }\end{array}$} \\
\hline $\begin{array}{l}\mathrm{F} \\
\mathrm{M} \\
\text { Median age, y (range) } \\
\text { HIV positive }\end{array}$ & $\begin{array}{c}115(58) \\
82(42) \\
51(0.8-92) \\
8(4)\end{array}$ & $\begin{array}{c}19(33) \\
38(67) \\
41(1-82) \\
34(60)\end{array}$ & $\begin{array}{c}15(54) \\
13(46) \\
44(0.8-76) \\
5(18)\end{array}$ & $\begin{array}{c}7(50) \\
7(50) \\
70(39-88) \\
0(0)\end{array}$ & $\begin{array}{c}20(52) \\
18(48) \\
61(21-88) \\
3(8)\end{array}$ & $\begin{array}{c}176(53) \\
158(47) \\
51(0.8-92) \\
50(15) \\
\end{array}$ \\
\hline \multicolumn{7}{|l|}{ Mycobacterium species } \\
\hline $\begin{array}{l}\text { Rapid-growing species } \\
\text { M. chelonae/abscessus complex } \\
\text { M. fortuitum complex } \\
\text { M. chelonae }\end{array}$ & $\begin{array}{l}85(43.1) \\
40(20.3) \\
28(14.2) \\
17(8.6)\end{array}$ & $\begin{array}{l}5(8.8) \\
2(3.5) \\
1(1.8) \\
2(3.5)\end{array}$ & $\begin{array}{l}2(7.1) \\
1(3.6) \\
1(3.6) \\
0(0)\end{array}$ & $\begin{array}{l}3(21.4) \\
1(7.1) \\
1(7.1) \\
1(7.1)\end{array}$ & $\begin{array}{l}13(34.2) \\
8(21.1) \\
1(2.6) \\
4(10.5)\end{array}$ & $\begin{array}{l}108(32.3) \\
52(15.6) \\
32(9.6) \\
24(7.2)\end{array}$ \\
\hline $\begin{array}{l}\text { Slow-growing species } \\
\text { M. avium/intracellulare complex } \\
\text { M. marinum } \\
\text { M. goodii } \\
\text { M. aubagnense } \\
\text { M. xenopi } \\
\text { M. alvei } \\
\text { M. neoaurum } \\
\text { Other species } \neq\end{array}$ & $\begin{array}{c}70(35.5) \\
20(10.2) \\
5(2.5) \\
1(0.5) \\
1(0.5) \\
2(1) \\
0(0) \\
13(6.6)\end{array}$ & $\begin{array}{c}45(78.9) \\
0(0) \\
0(0) \\
1(1.8) \\
0(0) \\
0(0) \\
2(3.5) \\
4(7)\end{array}$ & $\begin{array}{c}23(82.1) \\
0(0) \\
1(3.6) \\
0(0) \\
1(3.6) \\
0(0) \\
0(0) \\
1(3.6)\end{array}$ & $\begin{array}{c}9(64.3) \\
0(0) \\
0(0) \\
0(0) \\
0(0) \\
0(0) \\
0(0) \\
2(14.3)\end{array}$ & $\begin{array}{c}20(52.6) \\
1(2.6) \\
0(0) \\
1(2.6) \\
1(2.6) \\
0(0) \\
0(0) \\
2(5.3)\end{array}$ & $\begin{array}{c}167(50) \\
21(6.3) \\
6(1.8) \\
3(0.9) \\
3(0.9) \\
2(0.6) \\
2(0.6) \\
22(6.6)\end{array}$ \\
\hline
\end{tabular}

*Values are no. (\%) patients except as indicated. Total excludes 13 M. gordonae isolates: 6 skin/soft tissue, 1 disseminated, 1 lymph node, 5 other. †Skin/soft tissue: wound, abscess, tissue, or exit catheter; disseminated: blood, bone marrow, cerebrospinal fluid, pericardial fluid, or peritoneal fluid; lymph node: lymph node or neck abscess; joint: synovial or joint fluid; other: urine, eye, sinus, or nasopharyngeal.

†Other species were 8 unspeciated and 1 each of $M$. asiaticum, M. branderi, M. brisbanense, M. heckeshornense, M. immunogenum, M. interjectum, M. kansasii, M. lentiflavum, M. Ilaterense, M. mucogenicum/phocaicum, M. obuense, M. simiae, M. thermoresistible, and M. vaccae. 


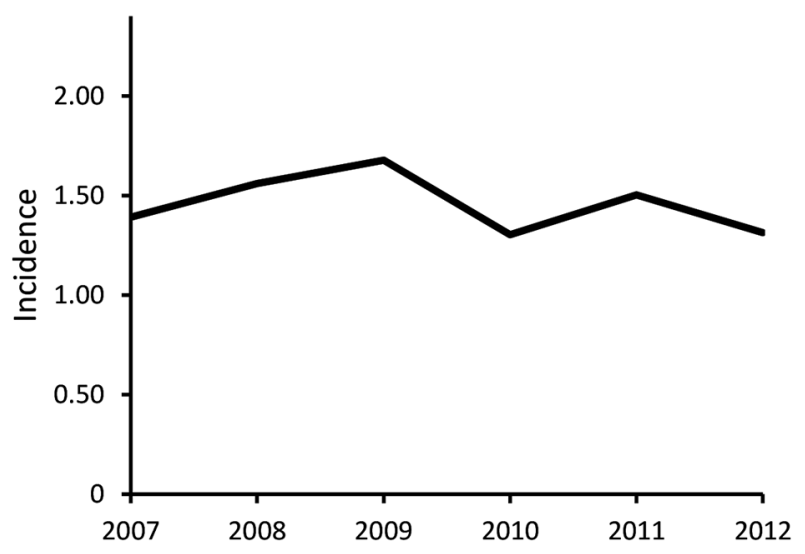

Figure. Observed incidence (cases/100,000 population) of extrapulmonary nontuberculous mycobacterium infection (excluding Mycobacterium gordonae), Oregon, USA, 2007-2012.

M. abscessus/chelonae complex, and 0.1/100,000 each for M. fortuitum, M. chelonae, and M. marinum.

\section{Discussion}

We describe Oregon's population-based experience with extrapulmonary NTM infections before these infections were made reportable in 2014. The annual incidence remained stable over the study period, averaging 1.5 cases/100,000 population. In contrast with pulmonary NTM disease, which occurs predominantly in female patients, half of patients with extrapulmonary infections and two thirds of patients with disseminated NTM are male $(8,9)$. A smaller proportion of extrapulmonary infections is caused by MAC than by pulmonary disease, and RGM cause $43.1 \%$ of skin/soft tissue infections. Overall, $15 \%$ of extrapulmonary infections occurred in HIV-positive patients.

Our observed overall average incidence was comparable to the annualized prevalence ( 1.6 cases $/ 100,000$ population) originally reported in Oregon during 20052006 (10). The skin/soft tissue prevalence/incidence in each time period was also identical at 0.9 cases $/ 100,000$ population, accounting for $58.9 \%$ of all extrapulmonary infections in our study. More recently, Smith et al. reported a higher prevalence of extrapulmonary NTM infection of 2.8 cases $/ 100,000$ population in 3 counties in North Carolina during 2006-2010 (11). Of 184 North Carolina patients with non- $M$. gordonae extrapulmonary NTM isolates, $51(28 \%)$ were from a sterile site (equivalent to joint/disseminated/lymph node by our definition), $15(8 \%)$ were dermal, $7(4 \%)$ were catheter/implant related, and $111(60 \%)$ were categorized as other. Because of their different classifications, it is difficult to compare results by site directly. However, in North Carolina, a similar proportion of extrapulmonary infections overall was caused by rapidly growing NTM (37\%, compared with $31 \%$ in our study).
RGM are most commonly associated with skin/soft tissue infections. We observed a similar proportion of skin/soft tissue infections caused by RGM in Oregon compared with our prior study ( $43 \%$ vs. $51 \%$ in $2005-$ 2006). Although describing small subsets of our skin/ soft tissue infection category, a similarly or slightly higher proportion of dermal $(10 / 15,67 \%)$ and catheter/ implant-related $(2 / 7,43 \%)$ extrapulmonary infections in North Carolina were caused by RGM (11). In our data, $>80 \%$ of RGM $M$. fortuitum and slow-growing $M$. goodii and M. marinum infections were associated with skin/soft tissue infections.

Other categories of extrapulmonary NTM infections were less common. Disseminated infection, representing $17 \%$ of extrapulmonary infections, typically occurs in severely immunocompromised patients with AIDS (CD4+ counts $<50$ ), hematologic malignancies, or transplants $(12,13)$. Positive HIV status was a notable contributor to infection in our study, associated with $60 \%$ of disseminated NTM. As reported previously, median annual incidence of disseminated NTM in Oregon during 2007-2012 in HIVpositive patients was high, at 110 cases/100,000 HIV person-years (13). Given the lower proportion of lymph, skin/ soft tissue, or other infections with HIV, it is possible that some of these infections also represent disseminated infection in HIV patients.

In our data, only $43 \%$ of patients with pediatric lymphadenitis were $<5$ years of age, even after excluding those with HIV. Pediatric lymphadenitis occurs primarily in immunocompetent children $<4$ years of age, so this is an unusual pattern (14). Lymph node infections represented $<10 \%$ of extrapulmonary infections. In the North Carolina study, only $3 \%$ of cases were isolated from the lymph node, although some may have been misclassified as neck infections (11). Of the 12 neck isolations in North Carolina, 8 were in children $\leq 3$ years of age. In contrast, patients in our study with NTM infections of the joint ( $4 \%$ of total extrapulmonary infections), with a median age of 70, likely represent surgical site infections. Oregon previously investigated a cluster of 9 NTM infections involving joint prostheses occurring in 2013-2014 (15).

The strengths of this study include complete capture of extrapulmonary cases statewide over a 7-year period, allowing population-based analyses and analysis of trends. The disease incidence should be considered a minimal estimate, requiring the physician to order the appropriate diagnostic test (acid-fast bacillus culture). We were also able to link to the state HIV database and identify HIV-positive patients. Study limitations included a lack of clinical information to identify other underlying conditions and risk factors for infection. We were also unable to distinguish $M$. chelonae from M. abscessus in the M. chelonaelabscessus complex cases. 
More detailed clinical data and exposure history for NTM infections in Oregon will be available in the future from state surveillance efforts, aiding in the identification of outbreaks that require public health intervention. However, it is likely that relatively few cases are associated with outbreaks. During the first 2 years of reportability in Oregon, only $11(11 \%)$ of 98 extrapulmonary NTM isolates were linked epidemiologically (16). Reporting and follow-up of all patients with extrapulmonary isolates may be useful for detecting previously unidentified environmental sources of NTM, such as specific watersheds. Subspeciation and molecular typing of isolates may be necessary to identify clusters of more common species. Detailed follow-up on patients isolating M. gordonae to confirm whether it is the most likely cause of disease will inform whether or not to include it as a reportable infection along with other NTM species.

In conclusion, unlike pulmonary infections, which are increasing, extrapulmonary NTM incidence in Oregon is stable. Similar to pulmonary NTM, MAC causes most disseminated and lymph node infections. In contrast, RGM species are much more common causes of skin/soft tissue and other infections. Although the literature highlights clusters and outbreaks, most extrapulmonary NTM infections are likely isolated cases. Now that extrapulmonary NTM infections have been made reportable in Oregon (2014) and other states, we anticipate additional population-based estimates to be made available in the future.

This study was supported by the University of Rochester Respiratory Pathogens Research Center/National Institute of Allergy and Infectious Disease (HHSN272201200005C).

E.H. contributed to the acquisition, analysis, and interpretation of data, and developed the manuscript; S.S., K.H., and K.L.W. contributed to the concept and study design, interpretation of data, and reviewing the manuscript critically for intellectual content.

Dr. Henkle is a research assistant professor and infectious disease epidemiologist at Oregon Health and Science UniversityPortland State University School of Public Health in Portland, Oregon, USA. Her research interests include the quantitative and qualitative burden of pulmonary NTM infection and other respiratory diseases.

\section{References}

1. Lyman MM, Grigg C, Kinsey CB, Keckler MS, Moulton-Meissner H, Cooper E, et al. Invasive nontuberculous mycobacterial infections among cardiothoracic surgical patients exposed to heater-cooler devices. Emerg Infect Dis. 2017;23:796-805. http://dx.doi.org/ 10.3201/eid2305.161899

2. Schnabel D, Esposito DH, Gaines J, Ridpath A, Barry MA, Feldman KA, et al.; RGM Outbreak Investigation Team. Multistate US outbreak of rapidly growing mycobacterial infections associated with medical tourism to the Dominican Republic, 2013-2014. Emerg Infect Dis. 2016;22:1340-7. http://dx.doi. org/10.3201/eid2208.151938
3. Winthrop KL, Abrams M, Yakrus M, Schwartz I, Ely J, Gillies D, et al. An outbreak of mycobacterial furunculosis associated with footbaths at a nail salon. N Engl J Med. 2002;346:1366-71 http://dx.doi.org/ 10.1056/NEJMoa012643

4. Griffith DE, Aksamit T, Brown-Elliott BA, Catanzaro A, Daley C, Gordin F, et al.; ATS Mycobacterial Diseases Subcommittee; American Thoracic Society; Infectious Disease Society of America. An official ATS/IDSA statement: diagnosis, treatment, and prevention of nontuberculous mycobacterial diseases. Am J Respir Crit Care Med. 2007;175:367-416. http://dx.doi.org/ 10.1164/ recm.200604-571ST

5. Henkle E, Hedberg K, Schafer S, Novosad S, Winthrop KL. Population-based incidence of pulmonary nontuberculous mycobacterial disease in Oregon 2007 to 2012. Ann Am Thorac Soc. 2015;12:642-7. http://dx.doi.org/ 10.1513/AnnalsATS.201412-559OC

6. Marras TK, Mendelson D, Marchand-Austin A, May K, Jamieson FB. Pulmonary nontuberculous mycobacterial disease, Ontario, Canada, 1998-2010. Emerg Infect Dis. 2013;19:1889-91. http://dx.doi.org/10.3201/eid1911.130737

7. Portland State University, College of Urban and Public Affairs Population Research Center. Population estimates [cited 2013 Sep 5]. http://www.pdx.edu/prc/population-estimates-0

8. Prevots DR, Shaw PA, Strickland D, et al. Nontuberculous mycobacterial lung disease prevalence at four integrated health care delivery systems. American journal of respiratory and critical care medicine 2010;182:970-6.

9. Winthrop KL, McNelley E, Kendall B, et al. Pulmonary nontuberculous mycobacterial disease prevalence and clinical features: an emerging public health disease. American journal of respiratory and critical care medicine 2010;182:977-82.

10. Cassidy PM, Hedberg K, Saulson A, McNelly E, Winthrop KL. Nontuberculous mycobacterial disease prevalence and risk factors: a changing epidemiology. Clin Infect Dis. 2009;49:e124-9. http://dx.doi.org/ 10.1086/648443

11. Smith GS, Ghio AJ, Stout JE, Messier KP, Hudgens EE, Murphy MS, et al. Epidemiology of nontuberculous mycobacteria isolations among central North Carolina residents, 2006-2010. J Infect. 2016;72:67886. http://dx.doi.org/10.1016/j.jinf.2016.03.008

12. Henkle E, Winthrop KL. Nontuberculous mycobacteria infections in immunosuppressed hosts. Clin Chest Med. 2015;36:91-9. http://dx.doi.org/10.1016/j.ccm.2014.11.002

13. Varley CD, Ku JH, Henkle E, Schafer SD, Winthrop KL. Disseminated nontuberculous mycobacteria in HIV-infected patients, Oregon, USA, 2007-2012. Emerg Infect Dis. 2017;23:533-5. http://dx.doi.org/10.3201/eid2303.161708

14. Zimmermann P, Tebruegge M, Curtis N, Ritz N. The management of non-tuberculous cervicofacial lymphadenitis in children: a systematic review and meta-analysis. J Infect. 2015;71:9-18. http://dx.doi.org/10.1016/j.jinf.2015.02.010

15. Buser G, Laidler M, Beldavs Z, Cieslak P. Transient human colonization and substandard infection control practices associated with non-tuberculous mycobacteria surgical site infections Oregon, 2010-2014. Presented at: Council of State and Territorial Epidemiologists Annual Conference; Boston, MA, USA; June 14-18, 2015.

16. Oregon Health Authority, Public Health Division. Selected reportable communicable disease summary 2015 [cited 2017 Jul 17]. http://www.oregon.gov/OHA/PH/DISEASESCONDITIONS/ COMMUNICABLEDISEASE/DISEASESURVEILLANCEDATA/ ANNUALREPORTS/Documents/2015/arpt15.pdf

Address for correspondence: Emily Henkle, OHSU-PSU School of Public Health, 3181 SW Sam Jackson Park Rd, GH104, Portland, OR 97239, USA; email: emhenkle@gmail.com 